\title{
Innovación y Emprendimiento, y su relación con el Desarrollo Local del Pueblo de Salinas de Guaranda, Provincia Bolívar, Ecuador
}

\author{
Milton C. Barragán ${ }^{(1)}$ y Victor D. Ayaviri(2) \\ (1) Facultad de Recursos Naturales, Instituto de Posgrado y Educación Continua, Escuela Superior \\ Politécnica de Chimborazo, Riobamba-Ecuador (e-mail: mcbarragan@pronaca.com) \\ (2) Facultad de Ciencias Políticas y Administrativas, Universidad Nacional de Chimborazo, Riobamba- \\ Ecuador (e-mail: vdayaviri@gmail.com)
}

Recibido Mar. 15, 2017; Aceptado May. 17, 2017; Versión final Jul. 27, 2017, Publicado Dic. 2017

\begin{abstract}
Resumen
El objetivo de la presente investigación fue analizar empíricamente la relación entre innovación, emprendimiento y desarrollo local del pueblo de Salinas de Guaranda, provincia Bolívar, Ecuador. Para este propósito se empleo un enfoque cualitativo a través de la observación de campo y entrevistas, y otro cuantitativo con la aplicación de una encuesta que permita generar un modelo simple de regresión. Los resultados muestran que el emprendimiento contribuye de manera significativa al desarrollo local, no así la innovación. La organización comunitaria, la economía solidaria, liderazgo y cultura emprendedora son factores claves para el éxito de los emprendimientos en esta localidad. Se concluye que el emprendimiento ha contribuido al desarrollo local generando fuentes de empleo que permiten mejorar el nivel de vida de la poblacion basado en equidad, participación comunitaria y economía solidaria.
\end{abstract}

\section{Innovation and Entrepreneurship, and their Relation with Local Development in the Town of Salinas de Guaranda, Provincia Bolívar, Ecuador}

\begin{abstract}
The objective of the present research was to empirically analyze the relationship between innovation, entrepreneurship and local development of Salinas de Guaranda, Bolívar province, Ecuador. This study was divided in two parts: first a qualitative analysis carried on through field observations and interviews and secondly a quantitative analysis through the application of a survey, with the aim of developing a simple regression model. The findings show that entrepreneurship contributes significantly to local development, but not so innovation. Aspects such as community organization, solidarity economy, leadership and entrepreneurial culture, are the key factors for the success of entrepreneurship in this town. In conclusion, entrepreneurship has contributed to local development through generating employment to improve the living level of population, based on equity, community participation and solidarity economy.
\end{abstract}




\section{INTRODUCCION}

Las circunstancias económicas del mundo, la globalización, la proliferación de tratados comerciales, y la nueva sociedad del conocimiento, en apariencia, han puesto de moda la innovación. Sin embargo, realmente esta no es una temática reciente, ya que desde tiempo atrás se puede encontrar referentes teóricos que aluden a la innovación como pilar del desarrollo (Bustos, 2015).

En la perspectiva económica el desarrollo local requiere del crecimiento económico, a partir de ello, se puede incrementar el nivel de bienestar social y económico de una sociedad (Orozco y Nuñez, 2013), que se produce a partir de la aplicación de políticas públicas (Figueroa, 2012) y un bagaje de factores y estrategias que influyen en el desarrollo (Cruz, 2012). El desarrollo económico depende esencialmente de la capacidad para introducir innovaciones al interior de la base productiva y tejido empresarial de un territorio, y es preciso subrayar que la estrategia de desarrollo tiene su importancia en términos de empleo y territorio, basados en factores económicos, sociales, culturales y territoriales (Alburquerque, 2004). Por otra parte, el desarrollo genera espacios que permiten la transformación de los territorios, en tanto que el crecimiento está centrado en el incremento de la productividad e ingresos económicos (Álvarez y Alonso, 2005). Así, el desarrollo económico busca las condiciones necesarias para el desenvolvimiento de la sociedad a través de la participación, en procura de alcanzar escenarios de bienestar social y económico, que permitan garantizar los estándares mínimos de satisfacción de necesidades básicas de acceso a la educación, salud y empleo (Alburquerque, 2004).

El desarrollo local hace referencia a la capacidad que tiene una sociedad para formular objetivos de interés colectivo y movilizar los recursos necesarios para alcanzarlos y transformar su territorio (Alburquerque, 2004). Por lo tanto, el desarrollo local trata de una aproximación territorial, que hace referencia a los procesos de crecimiento y acumulación de capital de una localidad, que tiene cultura e instituciones que son propias y en las que se basan las decisiones de ahorro e inversión (Vázquez, 2007). Desde esta perspectiva, la política de desarrollo local constituye la respuesta de los actores y agentes locales a los desafíos de los procesos de transformación y cambios en los sistemas que producen el desarrollo (Vázquez, 2007), e implica por tanto, cambios en procesos sociales, políticos, ambientales y económico productivos (Amin y Robins, 1990; Cárdenas, 2002). Según Vázquez (2007) expresa que quizás el mayor atractivo del término desarrollo local sea su utilidad para interpretar los procesos de desarrollo de territorios y regiones, en tiempos donde se producen grandes transformaciones en la economía y la sociedad como consecuencia del aumento de la integración económica, política y cultural.

La cuestión no reside si el sistema productivo de una localidad o territorio está formado por empresas grandes o pequeñas, sino en el modelo de organización de la producción y sus efectos sobre el comportamiento de la productividad y de la competitividad (Vázquez, 2007). El desarrollo local busca incrementar las posibilidades de una sociedad aprovechando las potencialidades de los sujetos como iniciadores de ideas innovadoras que causan impacto económico y social a través del emprendimiento, posibilitando el crecimiento progresivo no solo en el empleo sino a nivel productivo, económico y social en las comunidades (Duarte y Ruiz, 2009). Así, la conceptualización del desarrollo local evoluciona y se transforma a medida que lo hace la sociedad y las ciudades, en la medida que las innovaciones y el conocimiento son difundidas, y éstas benefician al conjunto de la sociedad para su desarrollo ( Borea, 2006; Vázquez, 2007; Ulate, 2010), porque permite la diversificación de productos mejorados en base a la innovación (Marín y Rivera, 2014), fortalece la economía regional (Duarte y Ruiz, 2009; Curbelo y Peña, 2012; Schulz et al., 2017).

El impacto que generan los negocios ecuatorianos en cuanto a creación de empleo es bajo, tanto así que el $72.8 \%$ de los emprendimientos son unipersonales y con bajas expectativas de generación de empleo. Para los próximos 5 años casi un $31 \%$ de los negocios nacientes, $48 \%$ de los nuevos y $47 \%$ de los establecidos no esperan generar ninguna plaza de trabajo. Un $20.8 \%$ de la población adulta afirmó haber emprendido aprovechando una oportunidad, mientras que un $8.9 \%$ lo hizo por necesidad. Entre los emprendedores por oportunidad, el $22.5 \%$ lo hizo para mejorar ingresos actuales, el $35.2 \%$ para obtener mayor independencia y el $42.3 \%$ por ambos motivos (Lasio et al., 2016).

Por otro lado la fuerte migración rural urbana experimentada en las últimas décadas especialmente en países de América Latina, debido a la falta de oportunidades laborales, la baja calidad en la producción e ineficacia en la comercialización y la ineficiente gestión pública, generando una alta problemática socioeconómica, cultural y ambiental (Mata, 2014), como fue el caso de la parroquia Salinas de Guaranda en la década de los años setenta. Esta parroquia, con una poblacion cercana a los ocho mil habitantes, de los cuales el $60 \%$ son indígenas y $40 \%$ mestizos, viven en zonas geográficas que van desde los 600 hasta los 4200 metros de altitud con relación al nivel del mar. En esta región se observa un conjunto de iniciativas emprendedoras asociativas y unidades de negocios conformadas por pequeñas y medianas empresas con 
producción para el mercado local e internacional (Mata, 2014). Autores como Orejuela y Gordon (2015), explican que desde la década de los setenta, Salinas de Guaranda ha experimentado un proceso acelerado en el establecimiento y desarrollo de empresas, que coincide con la llegada de la misión salesiana y voluntarios de la organización Mato Grosso, quienes implantaron un modelo de desarrollo local basado en la organización, producción y comercialización que sustenta e impulsa la economía comunitaria. Salinas de Guaranda, cuya región fue considerada pobre, sin posibilidad de acceso a recursos productivos, y familias desempleadas, con una tasa de desocupación cercana al 50\%, debido a los efectos de las políticas capitalistas impulsadas por los gobiernos en la década de los años setenta, se enrumba hacia la sostenibilidad y economía solidaria a partir del año 1999, a través del cuidado del medio ambiente, el potenciamiento de los emprendimientos de turismo comunitario, comercio justo local e internacional, pasantías y transferencia de tecnología de campesino a campesino, la organización se abre al mundo y al comercio solidario integrando a todas sus organizaciones locales en este proceso para mejorar la calidad de vida de sus habitantes a través de los emprendimientos (Mata, 2014).

Con estos antecedentes y considerando como un caso de éxito a nivel nacional e internacional el desarrollo local de Salinas de Guaranda, el objetivo de la presente investigación fue determinar la relación existente entre innovación, emprendimiento y desarrollo local de Salinas a través de la observación de campo, entrevistas personales y la aplicación de encuestas a la poblacion objetivo para confirmar los aspectos cualitativos y cuantitativos de la investigación.

\section{MATERIALES Y METODOS}

La presente investigación corresponde a un trabajo no experimental bajo un diseño transversal de recolección de datos por medio de la observación de campo, entrevistas y la aplicación de una encuesta, asume el enfoque cualitativo y cuantitativo, en primera instancia se realiza la descripción del fenómeno de estudio y posteriormente un análisis cuantitativo y/o confirmatorio de los hallazgos del trabajo de campo que corresponde a una investigación de tipo descriptivo correlacional. La parroquia Salinas de Guaranda cuenta con 7262 habitantes, y el tamaño de la muestra para esta investigación corresponde a 356 personas, con un nivel de confianza del $95 \%$ y un nivel de significancia del $5 \%$. Para la aplicación de la encuesta como técnica de recolección de datos primarios se empleo como marco muestreo la cabecera parroquial, ya que es ahí donde los habitantes de esta parroquia convergen a realizar compras de víveres y tramites personales, se tomo en consideración la edad entre 11 y 60 años como variable demográfica para seleccionar los elementos muéstrales de la poblacion objetivo de donde se obtuvo la información de interés sobre la relación entre innovación, emprendimiento y desarrollo local. Se aplico un muestro probabilístico ya que las unidades de muestreo fueron seleccionadas al azar, a través de un muestro aleatorio simple, para garantizar que cada elemento de la poblacion objetivo tiene la misma probabilidad de ser incluida en la muestra.

Las técnicas de investigación cualitativa empleadas fueron la observación de campo a través de la visita a la parroquia de Salinas de Guaranda y a cada unas de las unidades de producción que tienen sus operaciones en la cabecera parroquial; y las entrevistas personales que se las desarrollo con los líderes de la parroquia, dirigentes de las unidades de producción, profesores de la unidad educativa Salinas y a productores campesinos que se encontraron el día de la entrevista en la cabecera parroquial.

Con la información levantada a través de la investigación cualitativa y cuantitativa se procedió a realizar análisis de los datos primarios con el uso de software estadístico SPSS, por sus siglas en ingles, que nos permitan aportar evidencias científicas para el cumplimiento del objetivo de la presente investigación.

\section{RESULTADOS}

El análisis de los resultados se desarrollo en dos etapas, en la primera se hace una descripción de la zona de estudio donde se llevo a cabo la investigación para luego realizar el análisis estadístico de los resultados obtenidos. En este sentido de acuerdo a Mballa (2016), para tener una compresión de los desafíos que enfrenta el desarrollo local es necesario incorporar en el análisis varios indicadores que abarcan, entre otros, las formas de gestión pública, el capital social, los esfuerzos asociativos, las relaciones económicas cooperativas y redes productivas, la pobreza, el rezago y la marginación social.

\section{i) Descripción de la zona de estudio}

Salinas de Guaranda está compuesta por la cabecera parroquial y treinta recintos aledaños, con una población cercana a los diez mil habitantes, en áreas geográficas que van desde los 600 hasta los 4.200 metros de altura sobre el nivel del mar en el centro de los Andes ecuatorianos entre los grandes volcanes Tungurahua y Chimborazo. Su cabecera parroquial, se localiza a 3.550 metros de altura sobre el nivel del 
mar con una población aproximada de mil habitantes. La convicción colectiva de salir de la pobreza en la que vivían los habitantes de Salinas de Guaranda ha sido un proceso de 4 décadas desde los años setenta, tiempo en el cual la organización solidaria y el compartir objetivos comunes han permitido lograr el tan anhelado buen vivir que hoy en día se basa en la equidad e igualdad de oportunidades para los miembros de una comunidad o colectivo (Mata, 2014).

Según Mata (2014), la cooperativa fue la única forma de asociación aplicable en Salinas en los años setenta y la primera herramienta de desarrollo comunitario en sus manos. La comunidad salinera estaba acostumbrada a realizar las cosas juntas bajo principios de reciprocidad y de ayuda mutua presentes desde siempre en su cultura. En estas circunstancias, sin factores productivos, con tasas de desempleo altas, en un sector tan vulnerable como el rural marginal de Salinas de Guaranda, en esa economía popular se tejían redes de solidaridad y tenían lugar diferentes experiencias y emprendimientos solidarios que no eran visualizados ni por el Estado ni por parte de la academia, estos emprendimientos no eran reconocidos por los analistas ni por los estudiosos e investigadores y, sin embargo, explicaban la manera de sobrevivir, la manera de subsistir y la manera de satisfacer las necesidades más básicas, construyendo procesos alternativos a la economía basada únicamente en el lucro (Mata, 2014). En la Tabla 1 se detalla el número de empresas establecidas en Salinas de Guaranda.

Tabla 1: Empresas establecidas en Salinas de Guaranda. (Datos tomados del PDOT GAD Salinas, 2015)

\begin{tabular}{|c|l|c|c|c|}
\hline \multirow{2}{*}{$N$ № } & \multicolumn{1}{|c|}{ Empresas } & No Empresas & No Empleados & \% Participación \\
\hline 1 & Cooperativa de Producción Agropecuaria el Salinerito (Lácteos) & 22 & 2118 & $74,68 \%$ \\
\hline 2 & Confites el Salinerito & 1 & 100 & $3,53 \%$ \\
\hline 3 & Embutidora Funorsal & 1 & 42 & $1,48 \%$ \\
\hline 4 & Fábrica de Conservas y Alimentos Salinerito & 1 & 45 & $1,59 \%$ \\
\hline 5 & Aceites Esenciales & 1 & 5 & $0,18 \%$ \\
\hline 6 & Hilandería Intercomunal Salinas & 1 & 90 & $3,17 \%$ \\
\hline 7 & Hotel el Refugio & 1 & 5 & $0,18 \%$ \\
\hline 8 & Asociación de mujeres artesanas de Salinas (Texal). & 1 & 137 & $4,83 \%$ \\
\hline 9 & Cooperativa de Ahorro y Crédito Salinas (Coacsal) & 1 & 16 & $0,56 \%$ \\
\hline 10 & Junta Parroquial Salinas & 1 & 26 & $0,92 \%$ \\
\hline 11 & Unidad Educativa del Milenio & 1 & 50 & $1,76 \%$ \\
\hline 12 & Restaurant & 3 & 6 & $0,21 \%$ \\
\hline 13 & Tiendas y panaderías & 6 & 11 & $0,39 \%$ \\
\hline 14 & Centros de computo & 2 & 2 & $0,07 \%$ \\
\hline 15 & Transporte Cooperado & NA & 120 & $2,22 \%$ \\
\hline 16 & Otros & 45 & 2836 & $100 \%$ \\
\hline
\end{tabular}

El $74,68 \%$ de los empleos generados en esta parroquia corresponden a la industria de procesamiento de lácteos con su amplia variedad de productos que se comercializan a nivel nacional e internacional, bajo la marca El Salinerito. A través de su centro de exportaciones también se comercializan confites, tejidos artesanales y materia prima con certificación orgánica como es caso del cacao fino de aroma.

\section{ii) Análisis estadístico}

El análisis cualitativo a través de la estadística descriptiva refleja que el 55,3\% de la población encuestada corresponde al género masculino y el 44,7\% corresponde al género femenino, con un promedio de edad de 23,87 años, el 67,4\% de familias de la localidad están constituidas por más de 4 personas; aspecto que se relaciona con el numero de 3,94 personas por hogar reportado por el INEC (2010), en el cantón Guaranda. Las fuentes de empleo contribuyen a generar ingresos para mejorar el nivel de vida de los habitantes de esta parroquia, ya que únicamente el 16,8\% de la poblacion encuestada indican que no disponen de un trabajo fijo, mientras que el $83,2 \%$ de las personas indican que por lo menos una persona de su hogar cuenta con una fuente de trabajo fijo. Estos valores se relacionan con lo expresado por Mata (2014), que indica que la mano de obra que absorbieron los emprendimientos "salineros" en el período de los años 2000 al 2007 se duplicó al pasar de doscientos trabajadores a una plantilla laboral de cuatrocientas personas, lo que representa un incremento de personal del $100 \%$. En este sentido el $68,9 \%$ de la poblacion indica que 
las principales actividades económicas que generan la mayor parte de ingresos son la agricultura y ganadería ya que son socios y proveedores de materia prima para la industria láctea de la parroquia que genera el $74,68 \%$ de los empleos a través del trabajo asociativo entre las diferentes comunidades. Así, la afirmación con la que parte el estudio, que el emprendimiento y la innovación contribuyen al desarrollo local de Salinas de Guaranda, se ratifica a través de los resultados descriptivos, que indican que el $91,1 \%$ de la poblacion coinciden en que los emprendimientos establecidos en la actualidad contribuyen al desarrollo local, puesto que generan importantes fuentes de empleo e ingresos, ya que el 38,9\% de la poblacion percibe ingresos mensuales mayores a 300 dólares por persona.

Como resultado de la observación de campo y entrevistas realizadas se enfatiza sobre la cultura emprendedora y trabajadora de los "salineros" que lo consideran como una identidad propia que se convierte en ventaja competitiva, se menciono por varias personas que se destina un fondo del $10 \%$ de los ingresos de cada unidad productiva al desarrollo de nuevos productos que son testeados en la ferias exposiciones en las que participa la empresa comercializadora para evaluar su aceptación por parte de los consumidores finales.

En este sentido la importancia de las industrias locales, radica en que crean empleo y generan recursos económicos para los involucrados (Oluwayemisi y Fillis, 2016). Con este enfoque Gumbau (2016) sostiene que existen regiones con diferentes capacidades socioeconómicas, y que estas regiones son más propicias a la innovación y al emprendimiento, como es el caso de Salinas de Guaranda, también se consideran como un activo endógeno al conjunto de emprendimientos que tiene un efecto positivo en los aspectos económicos, sociales y ecológicos en el desarrollo local (Waichung, 2014; Belz y Binder, 2015); hay que decir, sin embargo, que el éxito del desarrollo local se manifiesta claramente en casos en los cuales la colectividad dispone de recursos accesibles (Klein, 2005). En esta línea el 63,7\% de la poblacion expresan que el factor más relevante que ha contribuido al desarrollo local de Salinas de Guaranda, es el sistema de organización comunitaria y su gente. La innovación aun no es vista como elemento clave del desarrollo local ya que solamente el $3,2 \%$ de la poblacion lo considera importante. Los emprendimientos se encuentran concentrados en los sectores lácteos, artesanías, turismo comunitario, producción de chocolates, producción de mermeladas y embutidos entre los principales. Las inversiones locales van desde los 6.000 a 15.000 para implantar un negocio o emprendimiento, a partir de ello, los propietarios incrementan las inversiones en función de la rentabilidad del negocio. Los microcréditos también han apoyado de forma importante en este propósito de diversificación y expansión de la producción local ya que el $48,8 \%$ de los fondos invertidos en emprendimientos provienen de créditos de sus cooperativas. Esto último se relaciona con lo que menciona García et al., (2013), que el acceso a la financiación externa ha sido identificado como uno de los determinantes de la innovación.

Para contrastar la Hipótesis de trabajo $(\mathrm{H} 1)$, donde se indica que la innovación y el emprendimiento han contribuido al desarrollo local de Salinas de Guaranda, se ha seleccionado el análisis de regresión como el método estadístico para analizar las relaciones entre las variables innovación y emprendimiento, y su efecto en el desarrollo local de la parroquia. De acuerdo con la regresión lineal categórica, a través del uso del software estadísticos SPSS, los resultados se muestran en la Tabla 2. Con el análisis de regresión lineal realizado se demuestra con el coeficiente de determinación ajustado $\left(R^{2}\right)$, que el 9,9\% del desarrollo local de Salinas de Guaranda está determinado por la innovación y emprendimiento que se desarrolla en esta parroquia.

Tabla 2: Estadística de regresión

\begin{tabular}{|l|l|}
\hline Coeficiente de correlación múltiple & 0,329 \\
\hline Coeficiente de determinación $\mathrm{R}^{2}$ & 0,108 \\
\hline $\mathrm{R}^{2}$ ajustado & 0,099 \\
\hline Error Típico & 0,892 \\
\hline
\end{tabular}

Además indica que existe un efecto de regresión altamente significativo para de las variables referidas, dado que se obtuvo un $p<0,000$ (Tabla 3), el cual es menor que el nivel crítico de comparación $($ alpha $\leq 0,05$ ).

Tabla 3: Modelo de regresión

\begin{tabular}{|l|l|l|l|l|l|}
\cline { 2 - 6 } \multicolumn{1}{c|}{} & $\begin{array}{c}\text { Suma de } \\
\text { cuadrados }\end{array}$ & $\begin{array}{c}\text { Grados de } \\
\text { libertad }\end{array}$ & Media cuadrática & F & Sig. \\
\hline Regresión & 20,530 & 2 & 10,265 & 11,327 & 0,000 \\
\hline Residual & 169,470 & 187 & 0,906 & & \\
\hline Total & 190,000 & 189 & & & \\
\hline
\end{tabular}


Considerando que la variable innovación y emprendimiento explican solamente el 9,9\% del desarrollo local visible en la parroquia Salinas de Guaranda es necesario señalar que existen otros elementos que contribuyen al desarrollo local como son la cultura, identidad y responsabilidad hacia el trabajo, la asociatividad y la economía solidaria entre los más importantes referidos en las entrevistas realizadas a los líderes comunitarios. Estos resultados los podemos relacionar con lo expresado por varios autores como, Borea 2006, Ulate 2010, Buckland y Murillo 2014, que sostienen que la innovación genera la creación de empresas, junto con los emprendimientos que promueven la creación de empleo, competitividad, oportunidades y crecimiento económico en las regiones (Duarte y Ruiz, 2009; Curbelo y Peña, 2012).

Por último las principales razones que impulsan la innovación y el emprendimiento con recursos locales para generar desarrollo local de la poblacion de Salinas de Guaranda, está la necesidad de contar con más recursos económicos que les permitan acceder a una mejor calidad de vida, ser reconocidos en el contexto regional como una comunidad y población emprendedora que le posicione en el contexto nacional e internacional como un modelo de desarrollo local exitoso.

\section{DISCUSION}

El presente estudio tiene como objetivo determinar la relación existente entre innovación, emprendimiento y desarrollo local de la parroquia Salinas de Guaranda, en base a los resultados presentados se determino que el emprendimiento tiene un efecto significativo sobre el desarrollo local de la poblacion de esta parroquia, a pesar que su peso es bajo, ya que existen otros factores con mayor contribución. Esto se sustenta con la afirmación del $91,1 \%$ de la poblacion encuestada que indica que los emprendimientos establecidos actualmente en Salinas de Guaranda contribuyen al desarrollo local a través de la creación de fuentes de empleo en las empresas establecidas en la parroquia, entre ellas la fábrica de producción de lácteos, confites y chocolates, la hilandería y tejidos, producción de artesanías, cooperativa de ahorro y crédito y turismo comunitario. En esta misma línea en el $83,2 \%$ de las familias por lo menos una persona cuenta con trabajo fijo en las unidades de producción, esto resalta el objetivo de bienestar social y de economía solidaria de los emprendimientos de esta poblacion que ha dejado de lado los intereses personales para salir de la pobreza extrema en que vivían en la década de los años setenta y hoy en día convertirse en un emporio de empresas. Estos resultados coinciden con lo expresado por Petit (2007), que indica que la innovación y el emprendimiento constituyen mecanismos que dinamizan recursos, capacidades y competencias para la generación de conocimiento que se plasma en estrategias para explotar nuevas oportunidades que económicamente generen valor. El emprendimiento rural se ha convertido en uno de los factores claves para el desarrollo local y la agroindustria (Wortman, 2009; Doherty, 2014). Las especificidades de las regiones son fundamentales para la ejecución de los proyectos; ya que los componentes de los ecosistemas de conocimiento e innovación son interdependientes y, como tales, deben actuar al unísono tanto en su estructura e influencia para materializar sus efectos sobre la economía y la sociedad a través de la innovación y el emprendimiento, generando transformaciones, cambios tecnológicos y mejoras en la competitividad (Osman, 2014; Spigel, 2017). Según Vázquez (2015), se dan estos fenómenos cuando existe un aumento de la productividad a través del incentivo a la producción local, la innovación y el emprendimiento como elementos importantes de los procesos de transformación y desarrollo. Por tanto, según Orozco y Nuñez (2013), indican que el desarrollo de una comunidad, es entendido como el cambio de una sociedad rural, agrícola, analfabeta, pobre y con crecimiento de población rápido, a otra que es principalmente, industrializada, como es el caso de Salinas de Guaranda.

De acuerdo a García et al., (2013), la innovación permite a las empresas lograr ventajas competitivas sostenibles en el tiempo y representa un elemento determinante del crecimiento económico de una poblacion. En Salinas de Guaranda únicamente el 3,2\% de la poblacion considera la innovación como importante. Esto se puede explicar ya que a través de la observación de campo y entrevistas realizadas se pudo constatar que no está claro el concepto de innovación, y se lo confunde muy fácilmente con emprendimiento y mejora continua de procesos. Se menciona además que los centros de producción establecidos en Salinas destinan el $10 \%$ de los ingresos para actividades de innovación y desarrollo de productos sin constatar en la práctica que exista un procedimiento formal para este tipo de iniciativas que permitan evaluar su eficacia a través del incremento de las ventas. En este contexto donde la innovación es alimentada por el conocimiento aplicado localmente, los emprendedores pueden convertir este conocimiento en innovación y contribuir al crecimiento local y regional, como sugiere la teoría del desborde de conocimiento (Capelo y Lenzi, 2016). Por otra parte, la innovación es ampliamente considerada como un motor clave del crecimiento económico, aunque las dimensiones sociales y culturales a menudo se descuidan; por aquello la innovación convergente combina la innovación tecnológica y social, con un enfoque especial en las poblaciones más desatendidas (Dargan y Shucksmith, 2008; Dube et al., 2014).Así Moreno et al., (2011), indican que el capital humano, es decir, el conjunto de conocimientos, destrezas y habilidades que tienen y utilizan los trabajadores influye positivamente en la innovación. 
El $63,7 \%$ de la poblacion considera que los principales factores de éxito de los emprendimientos actuales son la organización comunitaria y la actitud emprendedora de su gente, esto lo vivieron en la práctica cuando se trato de replicar este modelo de desarrollo en otras localidades de la provincia Bolívar, sin tener el éxito esperado ya que la cultura y liderazgo son muy diferentes a los "salineros" que vienen de un proceso de transformación de más de cuatro décadas. En este sentido Méndez (2004), señala que desde el punto de vista de los principios teóricos, el desarrollo local, en un entorno como el actual caracterizado por los cambios continuos en la tecnología, la economía y la sociedad requiere fomentar la innovación, la capacidad emprendedora, la calidad del capital humano, y la flexibilidad del sistema productivo. Lo que se complementa con lo expresado por Galindo et al., (2016), que indica que los emprendedores, gracias al empleo de sus propios recursos o de los que obtienen en el mercado y a las innovaciones que en ocasiones generan o introducen, posibilitan, junto a otros agentes sociales, la creación de la riqueza que luego se repartirá entre los distintos componentes de la sociedad.

No obstante, si bien los resultados permiten evidenciar que el emprendimiento contribuye de manera significativa al desarrollo local, la innovación no se ha convertido en un catalizador de proyectos de desarrollo para la comunidad, ya que existen otros factores que debieran ser analizados, como la cultura emprendedora y la inversión local de la población, en relación a otras regiones. Esto resultados se relacionan con lo expresado por Álvarez y Bernal (2017), que indican que el éxito de la innovación abierta en las empresas se da por la interacción de los siguientes factores clave: el potencial humano (eje del modelo), la cultura organizacional, la estrategia, la estructura organizacional, el uso de las tecnologías de la información y las herramientas propias de este tipo de innovación. Por ello, a manera de discusión se formula la siguiente interrogante: ¿en qué medida influye la cultura emprendedora de la comunidad en los emprendimientos, y cuál es la motivación que tienen para realizar inversiones locales?

\section{CONCLUSIONES}

A través de la revisión de literatura, el trabajo de campo y el análisis empírico, se comprueba que el emprendimiento contribuye de manera significativa al desarrollo local de Salinas de Guaranda, por medio de la creación de fuentes de empleo que generan ingresos para alcanzar un mejor nivel de vida de su poblacion.

Por otro lado la innovación no es considerada como un factor que ha contribuido al desarrollo local, ya que solo un porcentaje muy pequeño de la poblacion la considera importante. Esto se convierte en una gran oportunidad para la creación de un plan de innovación incremental y radical mediante procesos de capacitación del recurso humano que permitan incrementar los volúmenes de ventas de los emprendimientos a través del tiempo.

Los aspectos de organización comunitaria, economía solidaria, liderazgo y cultura emprendedora son considerados factores claves del éxito en los emprendimientos de Salinas de Guaranda, que han logrado convertirse en pequeñas y medianas empresas para alcanzar el desarrollo local de su territorio y su poblacion.

\section{REFERENCIAS}

Alburquerque, F., El Enfoque del Desarrollo Económico Local, Organización Internacional del Trabajo, ISBN: 92-2-316549-0, (1), 2-84 (2004)

Amin, A.; Robins, K., The Re-Emergence of Regional Economies The Mytical Geography of Flexible Accumulation, Environment and Planning, Society and Space, https://goo.gl/oyPjoP, (8), 7-34 (1990)

Álvarez, J.; Alonso, A., Nociones de Crecimiento y Desarrollo Económico, Revista Galega de Economía, ISSN: 1132-2799, goo.gl/yEheGS, 15(2), 1- 10 (2005)

Álvarez, E.; Bernal, C., Modelo de Innovación Abierta: Énfasis en el Potencial Humano, doi: 10.4067/S071807642017000100007, Información Tecnológica, 28 (1), 65-76 (2017)

Belz, F.; Binder, J., Sustainable Entrepreneurship: A Convergent Process Model, Wiley Online Library, https://goo.gl/XGyXjV, (1), 1- 17 (2015)

Borea, F., Innovación y Desarrollo Económico, Hologramática, ISSN: 1668-5024, goo.gl/CtMc8V, 2(4), 1-19 (2006)

Buckland, H.; Murillo, D., La Innovación Social en América Latina, Instituto de Innovación Social, ISBN: $978-$ 84-697-1730-1, goo.gl/enXDqj, (75), 1-71 (2014) 
Bustos, O., La Innovación basada en Ciencia como Pilar de Desarrollo, El Mercurio, Ediciones Especiales, goo.gl/GTs1Jr (2015)

Cárdenas, N., El Desarrollo Local su Conceptualización y Procesos, Red de Revistas Científicas de América Latina, El Caribe, España y Portugal, ISSN: 1317-9535, https://goo.gl/Jdo3dK, (8), 53-76 (2002)

Capelo, R.; Lenzi, C., Innovation Models and Entrepreneurial Behavioral Characteristics in Regional Growth, Built Environment and Construction Engineering, (1), 1-19 (2016)

Cruz, B., Estrategias de Políticas Públicas para el Desarrollo Sustentable, una Visión Crítica, Revista de Estudios Interdisciplinarios en Ciencias Sociales, Universidad Rafael Belloso Chacín, ISSN: 1317-0570, Depósito legal pp: 199702ZU31, https://goo.gl/EJnE9i, 14(3), 346-363 (2012)

Curbelo, J. L.; Peña, I., Emprendimiento y Competitividad Regional, Boletín de Estudios Económicos, ISSN: 0006-6249, goo.gl/UQnc7i, 67(205), 59-76 (2012)

Dargan, L.; Shucksmith, M., LEADER and Innovation, European Society for Rural Sociology, goo.gl/hHVHQn, (3), 1-18 (2008)

Doherty, B., Social Enterprises as Hybrid Organizations: A Review and Research Agenda, doi: 10.1111/ijmr.12028, International Journal of Management Reviews, (16), 417-436 (2014)

Dube, L. y otros nueve autores, Convergent Innovation for Sustainable Economic Growth and Affordable Universal Health Care: Innovating the Way we Innovate, doi: 10.1111/nyas.12548, Annals of the New York Academy of Sciences, (1), 119-141 (2014)

Duarte, T.; Ruiz, M., Emprendimiento, Una Opción para el Desarrollo, Scientia et Technica, ISSN: 01221701, https://goo.gl/ik1BJX, 3(43), 326-331 (2009)

Figueroa, N., El Desarrollo y las Políticas Públicas, Polis, Revista Latinoamericana, ISSN: 0718-6568, https://polis.revues.org/8580, (33), 12-15 (2012)

Galindo-Martín, M.A.; M.T. Méndez-Picazo, M.S. Castaño-Martínez, Crecimiento, Progreso Económico y Emprendimiento, http://dx.doi.org/10.1016/j.jik.2016.01.006, J. of Innovation \& Knowledge, (1) 62-68 (2016)

García, D., Financiación de la innovación en las Mypyme Latinoamericanas, Est. Gerenciales, (29), 12-16 (2013)

Gumbau, M., Entrepreneurship, Innovation and Regional Performance: Application for the Spanish Regions, Entrepreneurship, Regional Development, https://goo.gl/oUSNxC, 1-22 (2016)

Instituto Nacional de Estadísticas y Censos INEC (2010)

Klein, J., Iniciativa Local y Desarrollo: Respuesta Social a la Globalización Neoliberal, http://dx.doi.org/10.4067/S0250-71612005009400002, Revista Eure, 31(94), 25-39 (2005)

Lasio, V. y otros tres autores, Global Entrepreneurship Monitor- Ecuador, Espae - Espol, ISSN: 13903047, https://goo.gl/ApfrLS, 1-82 (2016)

Mata, O., Los Proyectos Solidarios de Salinas de Guaranda y su Aporte para la Construcción de "Otra Economía". Tesis de Maestría, Facultad Latinoamericana de Ciencias Sociales, Ecuador, Biblioteca Digital de Vanguardia, para la Investigación en Ciencias Sociales, Región Andina y América Latina, goo.gl/LYq3xT (2014)

Marín, A.; Rivera, I., Revisión Teórica y Propuesta de Estudio sobre el Emprendimiento Social y la Innovación Tecnológica, doi: 10.15174/au.2014.708, Multidisciplinary Scientific Journal, (24) 1-11 (2014)

Méndez, E., Como Medir el Desarrollo Local según la Experiencia Cubana. Observatorio de Economía Latinoamericana, ISSN: 1696-8352, https://goo.gl/9WKF7X, (29) 1-19 (2004)

Mballa, D., Desarrollo Local y Micro Finanzas como Estrategias de Atención a las Necesidades Sociales: un Acercamiento Teórico Conceptual, Revista Mexicana de Ciencias Políticas y Sociales, ISSN: 2448-492X, goo.gl/ZevUzp, (229), 101-128 (2016) 
Moreno, I.; Real, J.C.; de la Rosa, Ma. Dolores, La Incidencia del Capital Humano y la Cultura Emprendedora de la Innovación, doi:10.1016/j.cede.2010.09.001, Cuadernos de Economía y Dirección de Empresa, (14) 139-150 (2011)

Oluwayemisi, A.; Fillis, I., The Role of Handicraft micro Enterprises as a Catalyst for Youth Employment, http://dx.doi.org/10.1080/17510694.2016.1247628, Business School, 2, 1-17 (2016)

Orozco, J.; Nuñez, P., Las Teorías del Desarrollo, Revista Electrónica de las Sedes Regionales de la Universidad de Costa Rica, ISSN: 2215-2458, (27), 1-26 (2013)

Orejuela, M.; Gordon, D., Diseño de un Modelo de Gestión Integrada para las Microempresas del Grupo el Salinerito, Ingeniería en Gerencia y Liderazgo, (75), 1-93 (2015)

Osman, D., The State and Innovation an Analytical Framework, doi: 10.1111/muwo.12077, The London School of Economics, (1), 1-22 (2014)

Petit, E., La Gerencia Emprendedora Innovadora como Catalizador del Emprendimiento Económico, Revista de Ciencias Sociales, ISSN: 1315-9518, goo.gl/NdZjFT, (3), 1-3 (2007)

Spigel, B., The Relational Organization of Entrepreneurial Ecosystems, doi:10.1111/etap.12167, Baylor University, (1), 1-24 (2017)

Schulz, M.; Urbig, D. y Procher, V., The Role of Hybrid Entrepreneurship in Explaining Multiple Job Holders' Earnings Structure, Journal of Business Venturing Insights, goo.gl/tgZEcz, (7), 9-14 (2017)

Ulate, I., La Innovación que Potencia el Desarrollo, Revista Nacional de Administración, 1(2), 79-86, goo.gl/qtYvFD, Julio-Diciembre (2010)

Vázquez, M. A., El Desarrollo Regional, Estudios Sociales, goo.gl/UP7GKB, 23(46), 354-364 (2015)

Vázquez, A., Desarrollo Endógeno: Teorías y Políticas del Desarrollo Territorial, vazquezbarquero@uam.es, Investigaciones Regionales, (11), 182-210 (2007)

Waichung, H., Regional Development in the Global Economy: A Dynamic Perspective of Strategic Coupling in Global Production Networks, Regional Science Policy, goo.gl/8W4zRP, (1), 1-24 (2014)

Wortman, M. Rural Entrepreneurship Research: Integration into the Entrepreneurship Field, 10.1002/15206297(199007)6:4<329::AID-AGR2720060405>3.0.CO;2-N, College of Business Administration, lowa Satate University, (4), 329-344 (2009) 
